\title{
IMPORTÂNCIA DAS VARIÁVEIS ALTURA DOMINANTE E ALTURA TOTAL EM EQUAÇÕES HIPSOMÉTRICAS E VOLUMÉTRICAS ${ }^{1}$
}

\author{
Helio Garcia Leite ${ }^{2}$ e Valdir Carlos Lima de Andrade $^{3}$
}

\begin{abstract}
RESUMO - Este estudo foi conduzido, visando avaliar as seguintes hipóteses: a) equações hipsométricas devem ser obtidas por parcela, apenas em função do dap; e b) equações volumétricas devem ser locais, em função apenas do dap. Foram utilizados dados de quatro parcelas distribuídas ao acaso em um povoamento de eucalipto de 67,81 ha e de 50 parcelas distribuídas ao acaso em um povoamento de 121,3 ha, da mesma espécie. Em uma análise inicial, a fim de obter valores reais de altura total e de volume sólido, realizou-se uma cubagem rigorosa de todas as árvores das quatro parcelas. Foram utilizados, ainda, dados de 277 árvores cubadas rigorosamente nos povoamentos. Para avaliar a exatidão das estimativas e as hipóteses formuladas, empregou-se o procedimento estatístico proposto por Leite \& Oliveira (2002). Com base nos resultados, as hipóteses a e b foram rejeitadas a $1 \%$ de probabilidade. Concluiu-se, portanto, que em um inventário florestal a variável $H d$ deve ser considerada e que as equações volumétricas devem ser regionais.
\end{abstract}

Palavras-chave: Equação hipsométrica, equação volumétrica e inventário florestal.

\section{IMPORTANCE OF THE VARIABLES DOMINANT HEIGHT AND TOTAL HEIGHT IN HIPSOMETRIC AND VOLUMETRIC EQUATIONS}

\begin{abstract}
This study was carried out to evaluate the following hypotheses: a) hipsometric equations should be obtained by plot, only in function of the $d b h$ and $b$ ) volumetric equations should be local only in function of the dbh. Data of 4 and 50 plots were randomly distributed in a 67.81 ha and 121.3 ha eucalypt stand, respectively. A rigorous scaling of all the trees in the 4 plots was initially carried out to obtain total height and solid volume. Data of 277 trees rigorously scaled in the stand were also used. The statistical procedure proposed by Leite \& Oliveira (2002) was used to evaluate the accuracy of the estimates and hypotheses. Based on the results, hypotheses $a$ and $b$ were rejected at $1 \%$ probability. It was concluded that, in a forest inventory, the variable dominant height should be considered and the volumetric equations should be regional.
\end{abstract}

Key words: $\quad$ Hipsometric equation, volumetric equation and forest inventory.

\section{INTRODUÇÃO}

Estimativas de volume de madeira em inventários florestais incluem erros de amostragem e erros de nãoamostragem. Os erros de amostragem são controláveis, sendo dependentes, por exemplo, do método de amostragem e do tamanho da amostra. Por outro lado, os erros de não-amostragem são de difícil identificação. São várias as fontes deste segundo tipo de erro, dentre elas o uso de equações inadequadas para estimar a altura e o volume de árvores individuais.

Na totalização de parcelas de inventário florestal é comum o uso de equações volumétricas, correlacionando

1 Recebido para publicação em 14.5.2001.

Aceito para publicação em 12.5.2003.

2 Prof. do Departamento de Engenharia Florestal da Universidade Federal de Viçosa - UFV, 36571-000 Viçosa-MG, $<$ hgleite@ufv.br>. ${ }^{3}$ Eng. Florestal em Doutoramento na UFLA - Bolsista CAPES, Curso de Pós-Graduação em Engenharia Florestal, Caixa Postal 37, 37200-000 Lavras-MG, <vclandrade@mailcity.com> ou <vclandrade@hotmail.com>. 
o volume ( $V$ ) com o diâmetro (dap) e a altura total $(H t)$, cabendo mencionar o modelo de Schumacher \& Hall (1933). Contudo, na maioria das vezes, apenas algumas alturas são obtidas nas parcelas do inventário. Neste caso, uma equação é utilizada para estimar a altura das demais árvores. Em alguns casos, equações hipsométricas são obtidas individualmente por parcela, ao correlacionar $H t$ com o dap, conforme alguns exemplos de modelos estatísticos apresentados em Husch et al. (1972), Avery \& Burkhart (1983), Guimarães (1994) e Huang et al. (2000). Em outros, uma única equação é obtida para todas as parcelas do inventário, cabendo mencionar, o modelo: $\operatorname{Ln}\left(H t_{i}\right)=\beta_{0}+\beta_{1} \cdot\left(\text { dap }_{i}\right)^{-1}+\beta_{2} \cdot \operatorname{Ln}\left(H_{i}\right)+\operatorname{Ln}\left(\varepsilon_{i}\right)$ proposto por Campos et al. (1984), em que $H d$ é a altura dominante da parcela. Este modelo permite diferenciar a altura total de árvores de mesmo dap, em locais com capacidades produtivas diferentes. Portanto, são duas as alternativas para estimar a altura total de árvores que tiveram apenas o dap medido em parcelas de inventário: a) uso de relações funcionais do tipo $H t=f(d a p)$, por parcela; e b) uso de relações funcionais do tipo $H t=f($ dap,$H d)$, para um conjunto de parcelas. Além desse segundo tipo, podem ser empregadas relações como $H t=f($ dap $, H d, I)$, ou $H t=f($ dap $, I, S)$, sendo $S$ o índice de local e $I$ a idade. Exemplos são encontrados em Campos (1979), Campos (1986) e Leite (1990).

Por outro lado, o volume de árvores individuais pode ser obtido apenas em função do dap, empregando modelos locais que são relações funcionais do tipo $V=f($ dap $)$, conforme exemplos encontrados em Belchior (1996).

Essas alternativas de totalização de parcelas de inventário florestal têm sido utilizadas por diferentes empresas florestais, havendo ainda, em alguns casos, dúvidas sobre a importância da variável $H t$ na quantificação do volume e dúvidas sobre a importância da variável $H d$ na estimação da $H t$. Essas dúvidas são importantes porque, embora o tempo gasto com deslocamentos das equipes de campo seja o fator que mais contribui para o custo do inventário, a medição de $H t$ s contribui de modo expressivo. Diante destas considerações, idealizou-se este estudo com o propósito de avaliar as seguintes hipóteses:

$H o_{(l)}$ : equações de altura total devem ser obtidas utilizando apenas o dap como variável independente.

\section{Versus}

$H a_{(1)}:$ não $H o_{(I)}$.

R. Árvore, Viçosa-MG, v.27, n.3, p.301-310, 2003
$H o_{(2)}$ : equações volumétricas devem ser locais, empregando-se relações funcionais do tipo $V=f($ dap $)$.

Versus

$H a_{(2)}$ : não $H o_{(2)}$.

\section{MATERIAL E MÉTODOS}

Foram utilizados inicialmente dados de quatro parcelas circulares de $471,4 \mathrm{~m}^{2}$, distribuídas ao acaso em um povoamento do híbrido entre Eucalyptus grandis e Eucalyptus urophylla, em regime de alto fuste, de 67,81 ha, com espaçamento inicial de $3 \times 3 \mathrm{~m}$ e idade de 7 anos, localizado na região nordeste da Bahia. Em cada parcela foram medidos o dap de todas as árvores, a altura total das 15 primeiras árvores e a altura total de cinco árvores dominantes, resultando, portanto, em $15 \times 4=60$ pares de $d a p$ e $H t$ relacionados com a altura dominante média $(H d)$ de cada parcela ${ }^{1}$. Em seguida, todas as árvores dessas parcelas foram cubadas rigorosamente até um diâmetro comercial de 4,0 cm com casca, obtendo-se o volume real de madeira dessas parcelas. Foram utilizados, também, dados de outras 50 parcelas provenientes de outro povoamento, de 121,3 ha, com as mesmas características. Nessas parcelas, foram mensurados todos os daps e as Hts das 15 primeiras árvores. Utilizaram-se, ainda, dados de 277 árvores-amostra cubadas rigorosamente (Quadro 1).

Com os dados das 277 árvores cubadas foram ajustados os seguintes modelos:

$$
\begin{array}{r}
\operatorname{Ln}\left(V_{i}\right)=\beta_{0}+\beta_{1} \cdot \operatorname{Ln}\left(\operatorname{dap}_{i}\right)+\beta_{2} \cdot\left(\operatorname{dap}_{i}\right)^{-1}+\varepsilon_{i} \\
\text { Brenac (citado em Belchior, 1996). } \\
\operatorname{Ln}\left(V_{i}\right)=\beta_{0}+\beta_{1} \cdot \operatorname{Ln}\left(\operatorname{dap}_{i}\right)+\beta_{2} \cdot \operatorname{Ln}\left(H t_{i}\right)+\operatorname{Ln}\left(\varepsilon_{i}\right) \\
\text { Schumacher \& Hall (1933). }
\end{array}
$$

Um teste de identidade de modelos foi utilizado para verificar a necessidade de uso de equações hipsométricas específicas para cada parcela. Para este propósito, utilizando-se as 60 observações de dap e $H t$ das quatro parcelas, foram ajustados os seguintes modelos:

\footnotetext{
1 Como árvore dominante, considerou-se a árvore com fuste retilíneo, sadia, com dap maior do que o dap das demais árvores e com a copa recebendo luz direta na parte superior e lateralmente.
} 


$$
\begin{aligned}
& \begin{aligned}
\operatorname{Ln}(H t) & =\beta_{1} \cdot D_{1}+\beta_{2} \cdot D_{2}+\beta_{3} \cdot D_{3}+\beta_{4} \cdot D_{4} \\
+ & \frac{\beta_{5} \cdot D_{1}}{d a p}+\frac{\beta_{6} \cdot D_{2}}{d a p}+\frac{\beta_{7} \cdot D_{3}}{d a p}+\frac{\beta_{8} \cdot D_{4}}{d a p}+\varepsilon_{i} \\
\operatorname{Ln}(H t) & =\beta_{0}+\frac{\beta_{1}}{d a p}+\varepsilon_{i}
\end{aligned}
\end{aligned}
$$

em que

$\left[\begin{array}{llll}D_{1} & D_{2} & D_{3} & D_{4}\end{array}\right]=\left[\begin{array}{llll}1 & 0 & 0 & 0\end{array}\right]$ para a parcela $1 ;$

$\left[\begin{array}{llll}D_{1} & D_{2} & D_{3} & D_{4}\end{array}\right]=\left[\begin{array}{llll}0 & 1 & 0 & 0\end{array}\right]$ para a parcela $2 ;$

$\left[\begin{array}{llll}D_{1} & D_{2} & D_{3} & D_{4}\end{array}\right]=\left[\begin{array}{cccc}0 & 0 & 1 & 0\end{array}\right]$ para a parcela 3; e

$\left[\begin{array}{llll}D_{1} & D_{2} & D_{3} & D_{4}\end{array}\right]=\left[\begin{array}{llll}0 & 0 & 0 & 1\end{array}\right]$ para a parcela 4.

Após o ajuste dos modelos $\mathrm{O}$ e $w$, foi organizado o quadro de análise de variância, seguindo-se o layout apresentado em Regazzi \& Leite (1992). Em seguida, para testar a redução provocada na soma de quadrados da regressão, devido ao uso do modelo reduzido $w$, realizou-se o teste de identidade de modelos, calculandose a estatística:

$$
F_{(H o)}=\left[\frac{S Q \operatorname{Reg}(\Omega)-S Q \operatorname{Reg}(w)}{(H-1) p}\right]\left[\frac{S Q \operatorname{Res}(\Omega)}{N-H p}\right]^{-1},
$$

com distribuição $F_{H o}$ tendo $H-1 p$ e $N-H_{p}$ graus de liberdade, considerando um nível de significância de $1 \%$. em que $S Q \operatorname{Re} g(\Omega)=$ soma de quadrados de parâmetros do modelo completo; $S Q \operatorname{Reg}(\mathrm{w})=$ soma de quadrados de parâmetros do modelo reduzido; $S Q R$ es = soma de quadrados do resíduo do modelo completo; $H$ = número de modelos comparados; $P=$ número de parâmetros do modelo reduzido; e $N=$ número total de observações considerando os $H$ modelos.

De posse do resultado do teste de identidade de modelos, isto é, da decisão em utilizar ou o modelo completo ou o modelo reduzido para estimar as alturas totais das árvores, avaliou a hipótese $H o_{(l)}$ versus $H a_{(l)}$, adotando-se o procedimento:

a) estimou-se a altura total das 114 (174-60) árvores que não tiveram a sua altura utilizada no ajuste dos modelos hipsométricos com a equação selecionada, conforme decisão obtida pelo teste de identidade de

Quadro 1 - Distribuição diamétrica e hipsométrica da cubagem rigorosa de 277 árvores-amostra e de 174 árvores

\begin{tabular}{|c|c|c|c|c|c|c|c|c|c|c|c|c|c|}
\hline \multirow{2}{*}{$\begin{array}{c}\text { Classe } \\
\text { de } d a p \\
(\mathrm{~cm})\end{array}$} & \multicolumn{12}{|c|}{ Centro de Classe de Altura Total (m) } & \multirow{2}{*}{ Total } \\
\hline & 10 & 12 & 14 & 16 & 18 & 20 & 22 & 24 & 26 & 28 & 30 & 32 & \\
\hline 6 & $1(0)$ & $9(1)$ & $12(1)$ & $2(0)$ & & & & & & & & & $24(2)$ \\
\hline 8 & & $1(5)$ & $16(0)$ & $18(3)$ & $3(0)$ & & & & & & & & $38(8)$ \\
\hline 10 & & & $1(5)$ & $18(6)$ & $13(5)$ & $6(1)$ & $1(0)$ & & & & & & $39(17)$ \\
\hline 12 & & & $0(1)$ & $3(2)$ & $19(11)$ & $13(6)$ & $6(3)$ & $1(0)$ & $1(0)$ & & & & $43(23)$ \\
\hline 14 & & & & & $9(5)$ & $12(3)$ & $9(24)$ & $5(0)$ & $1(0)$ & & & & $36(32)$ \\
\hline 16 & & & & & $2(0)$ & $9(0)$ & $14(30)$ & $10(17)$ & $0(0)$ & $1(1)$ & & & $36(48)$ \\
\hline 18 & & & & & & $3(0)$ & $14(4)$ & $9(26)$ & $0(1)$ & & $1(0)$ & & $27(31)$ \\
\hline 20 & & & & & & & $2(0)$ & $10(9)$ & $7(3)$ & & & & $19(12)$ \\
\hline 22 & & & & & & $1(0)$ & & $3(1)$ & $4(0)$ & & & & $8(1)$ \\
\hline 24 & & & & & & & & & & $1(0)$ & $1(0)$ & $2(0)$ & $4(0)$ \\
\hline 26 & & & & & & & & & & & $1(0)$ & $2(0)$ & $3(0)$ \\
\hline Total & $1(0)$ & $10(6)$ & $29(7)$ & $41(11)$ & $46(21)$ & $44(10)$ & $46(61)$ & $38(53)$ & $13(4)$ & $2(1)$ & $3(0)$ & $4(0)$ & $277(174)$ \\
\hline
\end{tabular}
encontradas nas quatro parcelas estudadas

Table 1 - Distribution diametric and hipsometric distribution of the rigorous scaling of 277 sample-trees and 174 trees in the 4 studied plots

Número entre parênteses são de árvores cubadas nas 4 parcelas. 
modelos (modelo completo ou modelo reduzido). Também, utilizando os 60 pares $d a p$ e $H t$, obtidos nas quatro parcelas, juntamente com as alturas dominantes de cada parcela, foi ajustado o modelo: $\operatorname{Ln}\left(H_{t}\right)=$ $\beta_{0}+\beta_{1} \cdot\left(\text { dap }_{i}\right)^{-1}+\beta_{2} \cdot \operatorname{Ln}\left(H d_{i}\right)+\operatorname{Ln}\left(\varepsilon_{i}\right)$ (3). A equação resultante foi utilizada para estimar $H t$ das 114 árvores;

b) empregando o procedimento estatístico proposto por Leite \& Oliveira (2002), a 1\% de probabilidade, as estimativas de $H t$, obtidas com as equações hipsométricas selecionadas em $a$, foram comparadas com as alturas observadas $(\mathrm{n}=114)$, tendo sido avaliada a exatidão das estimativas de $H t$.

Para avaliar as hipóteses $\mathrm{Ho}_{(2)}$ versus $\mathrm{Ha}_{(2)}$, foram utilizadas as equações volumétricas oriundas do ajuste dos modelos 1 e 2 . Essas equações foram empregadas para totalizar as quatro parcelas pertencentes ao primeiro projeto, bem como as 50 parcelas do segundo projeto. Em seguida, procedeu-se à comparação do modelo $1 \mathrm{com}$ o 2 pelo teste $F$ de Snedecor, conforme apresentado em Graybill (1976). O critério de decisão para avaliar a hipótese $\mathrm{Ho}_{(2)}$ versus $\mathrm{Ha}_{(2)}$ foi:

a) $F$ não-significativo, $H o_{(2)}$ não foi rejeitada;

b) $F$ significativo, as estimativas obtidas com as equações oriundas dos modelos 1 e 2 foram comparadas com os volumes reais através do procedimento estatístico de Leite \& Oliveira (2002), a 1\% de probabilidade.
Neste caso, adotou-se a equação de melhor exatidão e distribuição mais uniforme de resíduos.

De modo complementar, os volumes obtidos nas quatro parcelas foram extrapolados para 1 ha e, em seguida, foram estimados os intervalos de confiança, tendo sido avaliada a exatidão das estimativas da média por hectare obtidas com as equações 1 e 2 .

\section{RESULTADOS E DISCUSSÃO}

As equações hipsométricas e volumétricas, obtidas conforme item 2, estão no Quadro 2. Cabe lembrar que os valores de $R^{2}$ não devem ser comparados entre si, principalmente o da equação 5 , que não inclui a constante $\beta_{0}$.

O resultado do teste de identidade de modelos, aplicado para verificar a necessidade de uso de equações $H t=f(d a p)$ por parcela (equação 5) em vez de uma única (equação 4), encontra-se no Quadro 3, que foi elaborado conforme apresentado em Regazzi \& Leite (1992). Constata-se, pela não-significância da estatística $F_{(H o)}$, que uma única equação hipsométrica pode ser adotada, isto é, a equação 4 pode ser usada para estimar as alturas totais das árvores nas quatro parcelas.

Portanto, com base no Quadro 3, para decidir sobre as hipóteses $\mathrm{Ho}_{(l)}$ ou $H a_{(l)}$, empregou-se o procedimento

Quadro 2 - Equações e estatísticas dos modelos adotados para descrever a relação hipsométrica e a relação volumétrica, nas quatro parcelas cubadas

Table 2 - Equations and statistics of the models adopted to describe the hipsometric and volumetric relationships in the 4 cubed plots

\begin{tabular}{|c|c|c|c|c|}
\hline Equação & $R^{2}$ & $\mathrm{CV} \%$ & Dados Utilizados & $\mathrm{n}^{\mathrm{o}}$ \\
\hline$\hat{H} t=e^{\left(3,604094-\frac{7,653112}{d a p}\right)}$ & 0,761 & 2,4 & $\begin{array}{l}60 \text { pares de dap e Ht obtidos } \\
\text { nas quatro parcelas. }\end{array}$ & $4 *$ \\
\hline$\hat{H} t=e^{\left(\begin{array}{l}3,589317 \cdot D_{1}+3,485358 \cdot D_{2}+3,789985 \cdot D_{3}+3,657533 \cdot D_{4}-\frac{7,264120 \cdot D_{1}}{d a p}-\frac{5,666051 \cdot D_{2}}{d a p} \\
\frac{10,797883 \cdot D_{3}}{d a p}-\frac{8,535809 \cdot D_{4}}{d a p}\end{array}\right)}$ & 0,999 & 2,3 & $\begin{array}{l}15 \text { pares de dap e Ht obtidos } \\
\text { nas quatro parcelas. }\end{array}$ & 5 \\
\hline$\hat{H} t=e^{\left(1,40095-\frac{7,678502}{d a p}+0,692821 \cdot \operatorname{Ln}(H d)\right)}$ & 0,773 & 2,3 & $\begin{array}{l}60 \text { pares de dap e Ht obtidos } \\
\text { nas quatro parcelas, incluindo } \\
\text { as alturas dominantes }(H d)\end{array}$ & 6 \\
\hline$\hat{V}=e^{[-10,648633+1,835865 \cdot \operatorname{Ln}(\text { dap })+1,254373 . \operatorname{Ln}(H t)]}$ & 0,993 & 3,2 & $\begin{array}{l}\text { Dados da cubagem de } 277 \\
\text { árvores. }\end{array}$ & $7 *$ \\
\hline$\hat{V}=e^{\left[-6,933544+2,021013 \cdot \operatorname{Ln}(d a p)-\frac{5,486070}{d a p}\right]}$ & 0,979 & 5,7 & $\begin{array}{l}\text { Dados da cubagem de } 277 \\
\text { árvores. }\end{array}$ & 8 \\
\hline
\end{tabular}

* equações obtidas por Leite \& Andrade (2002), em que $D_{i}=1$ e $D_{j},=0$ para a parcela $i ; \mathrm{i} \neq \mathrm{j}, \mathrm{i}=1$ ou 2 ou 3 ou 4 e $\mathrm{j}=1,2,3$ e 4 ; $R^{2}=$ coeficiente de determinação ajustado; e CV\% = coeficiente de variação.

R. Árvore, Viçosa-MG, v.27, n.3, p.301-310, 2003 
estatístico proposto por Leite \& Oliveira (2002), em que foi avaliada a exatidão das estimativas geradas pela equação 4 (modelo reduzido) e pela equação 6 (modelo de Campos et al., 1984). Os resultados estão no Quadro 4.

Nota-se que apesar de as estimativas de $H t$ obtidas com as duas equações, $H t_{(4)}$ e $H t_{(6)}$, terem sido diferentes das $H t$ observadas, $H t_{\text {real }}$, com base na exatidão ( $s_{\hat{Y Y} \%} \mathrm{e}$ EMP), o pesquisador poderia selecionar a equação 4 , isto é, $H t=f($ dap $)$, e a hipótese $H o_{(l)}$ ser aceita.

Apesar de o teste de identidade de modelos ter indicado que não é necessário utilizar modelos hipsométricos independentes em cada uma das quatro parcelas e de os resultados mostrados no Quadro 4 demonstrarem uma exatidão maior da equação 4 , pode-se, em uma grande área florestal, obter tendências indesejáveis nas estimativas hipsométricas de algumas parcelas. A relação $H t \mathrm{e}$ dap pode não ser a mesma nos diferentes locais e, conseqüentemente, pode ser mais adequado empregar uma equação própria de cada parcela (equação 5). Portanto, sugere-se que, ao adotar apenas o dap como variável independente em modelos hipsométricos, é importante avaliar

Quadro 3 - Análise de variância, adotando-se as quatro parcelas, com o teste da hipótese: Ho: as equações 4 e 5 são idênticas versus $H a$ : não $H o$

Table 3 - Variance analysis adopting the 4 plots with the hypothesis test: Ho: the equations 4 and 5 are identical versus $\mathrm{Ha}$ : non $\mathrm{Ho}$

\begin{tabular}{|l|c|r|r|r|}
\hline \multicolumn{1}{|c|}{ FV } & GL & \multicolumn{1}{|c|}{ SQ } & \multicolumn{1}{c|}{ QM } & $F(H o)$ \\
\hline Parâmetros $(\Omega)$ & 8 & 566,892 & & \\
Parâmetros $(w)$ & 2 & 566,815 & & \\
Redução $(H o)$ & 6 & 0,077 & 0,01275 & $2,816^{n s}$ \\
Resíduos & 52 & 0,235542 & 0,004529 & \\
\hline \multicolumn{1}{|c|}{ Total } & 60 & & & \\
\hline
\end{tabular}

$n s=$ não-significativo a $1 \%$. a exatidão proporcionada pelas equações geradas para cada parcela com a exatidão resultante do uso de uma única equação para o conjunto de parcelas do inventário florestal, utilizando o procedimento proposto.

Em grandes áreas, a altura dominante $(H d)$, quando usada como variável independente em modelos hipsométricos, pode contribuir significativamente para redução da soma de quadrado dos resíduos, principalmente por permitir representar diferentes capacidades produtivas dos locais onde se encontram as parcelas. Neste caso, as relações entre $H t$ e dap podem diferir entre parcelas localizadas em áreas boas, médias e ruins. De modo geral, pode ser mais prudente utilizar a variável $H d$ em modelos hipsométricos, isto é, adotar a equação 6 em substituição à equação 4. Para comprovar tal fato, foram utilizados dados de parcelas permanentes lançadas em um povoamento de 121,3 ha de um inventário florestal contínuo, conforme descrito no item 2 . Nesta área foi lançada uma amostra de 50 parcelas, de $471,4 \mathrm{~m}^{2}$, distribuídas em cinco talhões com diferentes capacidades produtivas. Foi mensurada a altura total de aproximadamente $35 \%$ das árvores e a altura total de cinco árvores dominantes em cada parcela, totalizando 967 indivíduos para o ajuste dos modelos $\mathrm{O}, w$ e 3 . Este procedimento foi necessário por causa do baixo número de parcelas empregadas anteriormente (quatro parcelas somente), pois estas quatro parcelas provavelmente não foram suficientes para representar possíveis variações na relação hipsométrica por causa das diferenças de capacidade produtiva (Figura 1).

Empregando-se os dados de 967 pares de dap e $H t$ e 50 valores de $H d$, das 50 parcelas, foram obtidas as equações:

$\operatorname{Ln} \hat{(H t})=1,921363+\left(\frac{-7,075871}{d a p}\right)+0,513418 .(H d)$

$\operatorname{com} R^{2}=0,876$

Quadro 4 - Resultados do procedimento estatístico proposto por Leite \& Oliveira (2002) na análise das equações 4 e 6 Table 4 - Results of the statistical procedure proposed by Leite $\mathcal{E}$ Oliveira (2002) in the analysis of the equations 4 and 6

\begin{tabular}{|c|c|c|c|c|c|c|c|}
\hline Comparação & $F$ & $t$ & $1-|\bar{e}|$ & $r_{\hat{Y} Y}$ & $s_{\hat{Y} Y \%}$ & EMP & Conclusão \\
\hline$H t_{\text {real }}$ X equação 4 & $3,092^{n s}$ & $2,200^{n s}$ & 0,982 & 0,914 & 6,91 & 1,752 & $H t_{\text {real }} \neq H t_{(4)}$ \\
$H t_{\text {real }}$ X equação 6 & $3,800^{n s}$ & $3,173^{*}$ & 0,975 & 0,908 & 7,15 & 2,536 & $H t_{\text {real }} \neq H t_{(6)}$ \\
\hline
\end{tabular}

\footnotetext{
${ }^{\mathrm{ns}}=$ não-significativo a $5 \% \mathrm{e} *=$ significativo a $5 \%$ de probabilidade.
} 

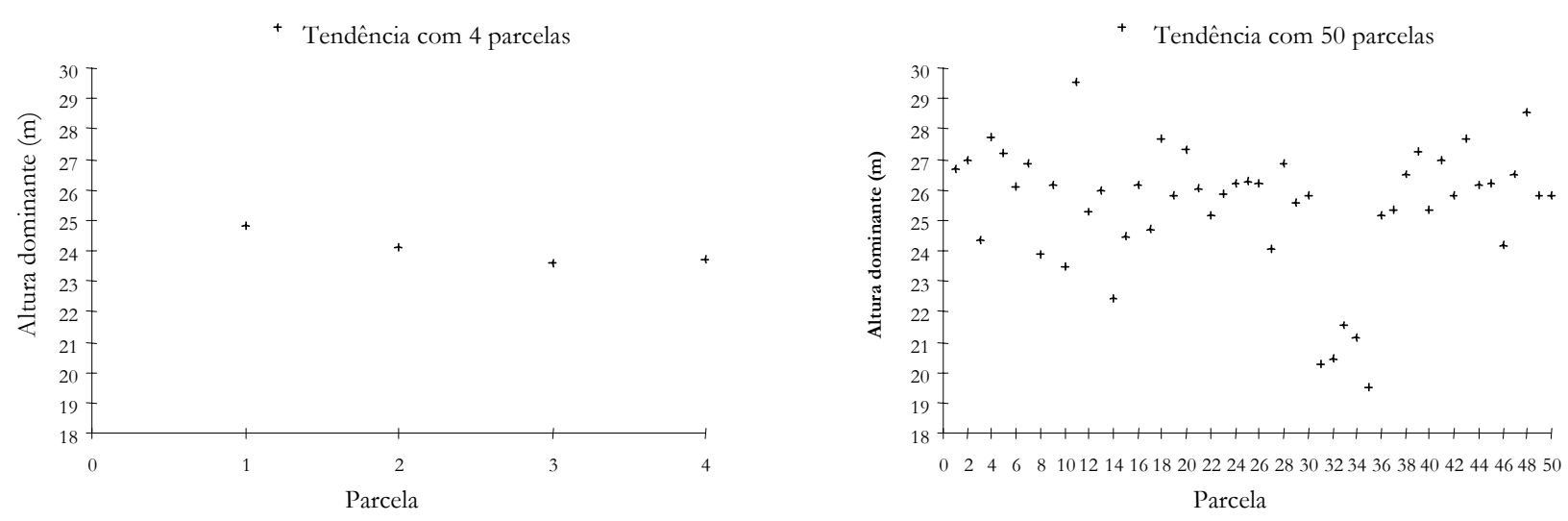

Figura 1 - Variação da altura dominante por parcela, utilizando dados de 4 e de 50 parcelas permanentes.

Figure 1 - Variation of the dominant height per plot using data of 4 and of 50 permanent plots.

$$
\operatorname{Ln} \hat{(H t})=3,588885+\left(\frac{-7,177247}{d a p}\right)
$$

com $R^{2}=0,833$ modelo $w$ e

$\operatorname{Ln} \hat{(H t})=\hat{\beta}_{1} \cdot D_{1}+\ldots+\hat{\beta}_{50} \cdot D_{50}+\left(\frac{\hat{\beta}_{51} \cdot D_{1}}{d a p}\right)+\ldots+\left(\frac{\hat{\beta}_{100} \cdot D_{50}}{d a p}\right)$

$\operatorname{com} R^{2}=0,894$, modelo $\Omega$.

No Quadro 5 está o resultado do teste de identidade de modelos utilizado para se verificar a necessidade de uso da equação 10 em vez da equação 11 . Constata-se, pela significância da estatística $F_{(H o)}$, que é necessário o uso de equações independentes para estimar as alturas totais das árvores das 50 parcelas, ou seja, não é correto adotar uma única equação hipsométrica para as 50 parcelas, aqui representada pela equação 10. É adequado, então, utilizar a equação 11. Conforme comentado, ao empregar modelos hipsométricos que utilizam somente o dap como variável independente, $H t=f(d a p)$, em grandes áreas florestais, é necessário o uso de equações independentes, e não uma única equação para todas as parcelas.

Os resultados mostrados no Quadro 6 indicam que as estimativas de $H t$, geradas pelas equações 9 e 11 , realmente diferem dos valores observados de $H t$, dado que a correlação foi inferior ao valor obtido por 1- $|\bar{e}|$, situação esta que, conforme Leite \& Oliveira (2002), indica diferença significativa, apesar de os testes $t$ e $F$ terem sido não-significativos. Entretanto, as estimativas de $H t$, geradas pela equação 11 , foram melhores que aquelas obtidas pela equação $9\left(<S_{\hat{Y Y} \%},>r_{\hat{Y Y} \%}\right)$. Para certificar-se desta superioridade, procedeu-se à análise gráfica de resíduos (Figura 2).

As estimativas obtidas de $H t$, empregando-se a equação 11 juntamente com a equação 9 , foram comparadas com os valores reais, adotando os mesmos procedimentos utilizados anteriormente, quando da análise das equações 4 e 6 . Os resultados estão no Quadro 6.

$\mathrm{Na}$ análise da Figura 2, constata-se que a tendência dos erros porcentuais mostrou-se mais uniforme e equilibrada para a equação 11 , porém esta superioridade não é expressiva a ponto de comprometer o uso da

Quadro 5 - Análise de variância, adotando as 50 parcelas, com o teste da hipótese: Ho: as equações 10 e 11 são idênticas versus $H a$ : não $H o$

Table 5 - Variance analysis adopting the 50 plots with the hypothesis test: Ho: the equations 10 and 11 are identical versus $\mathrm{Ha}$ : non $\mathrm{Ho}$

\begin{tabular}{|l|r|r|r|r|}
\hline \multicolumn{1}{|c|}{ FV } & \multicolumn{1}{|c|}{ GL } & \multicolumn{1}{c|}{ SQ } & QM & $F(H o)$ \\
\hline Parâmetros $(\Omega)$ & 100 & $9.124,741$ & & \\
Parâmetros $(w)$ & 2 & $9.121,608$ & & \\
Redução $(H o)$ & 98 & 3,13236 & 0,032 & $6,671^{*}$ \\
Resíduos & 867 & 4,154 & 0,005 & \\
\hline \multicolumn{1}{|c|}{ Total } & 967 & & & \\
\hline
\end{tabular}

* significativo a $1 \%$. 
Quadro 6 - Resultados do procedimento estatístico proposto por Leite \& Oliveira (2002) na análise das equações 9 e 11 Table 6 - Results of the statistical procedure proposed by Leite E Oliveira (2002) in the analysis of the equations 9 and 11

\begin{tabular}{|l|c|c|c|c|c|c|}
\hline \multicolumn{1}{|c|}{ Comparação } & $F$ & $t$ & $1-\mid \overline{\mathrm{e}}$ & $r_{\hat{Y} Y}$ & $s_{\hat{Y} Y \%}$ & EMP \\
\hline$H t_{\text {real }} \times$ Equação $9-H t=f($ dap,$H d)$ & $4,419^{\mathrm{ns}}$ & $1,132^{\mathrm{ns}}$ & 0,9971 & 0,937 & 6,681 & 0,290 \\
$H t_{\text {real }} \times$ Equação $11-H t=f($ dap $)$ & $1,199^{\mathrm{ns}}$ & $0,998^{\mathrm{ns}}$ & 0,9978 & 0,949 & 6,048 & 0,221 \\
\hline
\end{tabular}

ns $=$ não-significativo a $5 \%$ de probabilidade.

Equação 9

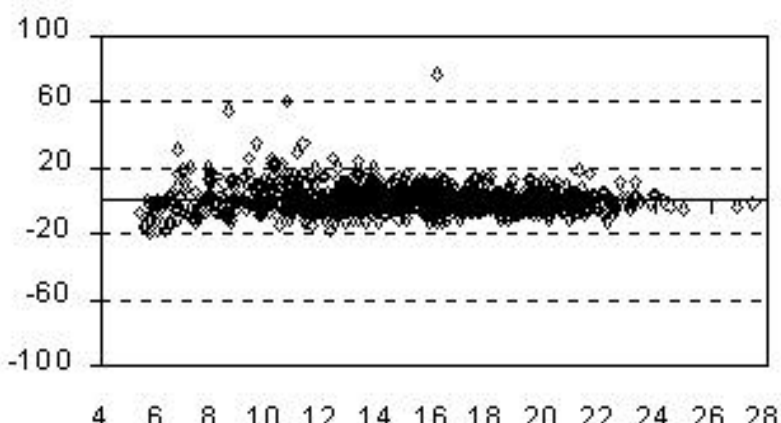

Equação 11

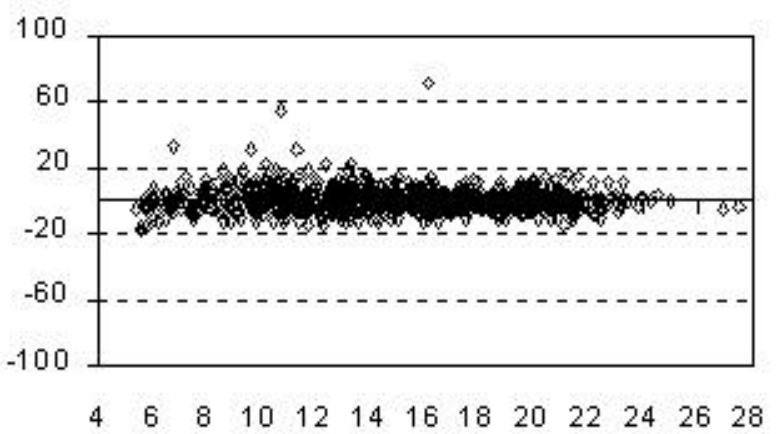

Figura 2 - Resíduos porcentuais em relação ao dap obtidos pelas equações 9 e 11 .

Figure 2 - Percentage residues based on the $\boldsymbol{d h h}$ obtained by the equations 9 and 11 .

equação 9. Aliás, os resultados obtidos por esta equação (Quadro 6) são aceitáveis e evidenciam o seu emprego na estimativa da altura total de árvores em grandes áreas florestais, além de também ser obtida uma desejável dispersão dos resíduos porcentuais (Figura 2). É feito apenas um ajuste com a equação 9, enquanto com a equação 11 são necessários tantos ajustes quantos forem os números de parcelas utilizadas na amostragem do inventário florestal.

O uso da equação 9 na estimativa da altura total em grandes maciços florestais, estatisticamente, tem o mesmo efeito da equação 11. É uma precaução contra a ocorrência e o efeito de diferentes capacidades produtivas sobre a relação Ht versus dap que não tenham sido representadas suficientemente pela estratificação realizada no inventário florestal. Neste caso, em grandes áreas, uma única relação hipsométrica do tipo $H t=f($ dap $)$ certamente não deve ser adotada, como também equações independentes, por ser um procedimento moroso (alto número de equações a ajustar) e por se ter resultados muito próximos daqueles obtidos com o modelo hipsométrico que tem $H d$.
Pode-se, então, inferir que a altura dominante é uma importante variável a ser incluída nos modelos hipsométricos. Além de contribuir de modo significativo para estimação da altura total, esta variável é geralmente utilizada nos métodos clássicos de classificação da capacidade produtiva de sítios florestais, necessária para o ajuste de muitos modelos de crescimento e produção. Ou seja, a variável $H d$ é geralmente obtida ao conduzir inventários contínuos e deve ser utilizada nas relações hipsométricas. Então, a hipótese $H o_{(1)}$ deve ser rejeitada.

Ao comparar os volumes obtidos pela equação 8, $V=f($ dap $)$, com aqueles obtidos pela equação 7 , $V=f(d a p, H t)$ e $H t=f(d a p)$, equação 4 para as árvores em que não foi feita a medição da altura total, conforme item 2 , obteve-se $F=81,889$ significativo a $1 \%$ de probabilidade, o que indica apenas que as estimativas de volume por árvore individual, obtidas em função do dap e em função do dap e de $H t$, são estatisticamente diferentes a $1 \%$, pelo teste $\mathrm{F}$ proposto por Graybill (1976). Para decidir sobre a hipótese $\mathrm{Ho}_{(2)}$, foi necessário avaliar a exatidão das estimativas geradas por estas equações (Quadro 7) e a distribuição dos resíduos (Figura 3). 
Ao analisar os resultados do Quadro 7, verifica-se que para as duas equações ( 7 e 8 ) ocorreram $F$ significativo, $r_{\hat{Y} Y}>1-|\bar{e}|$ e $t$ não-significativo. Aqui, cabe ressaltar que o teste $F$ proposto por Graybill pode se tornar inconsistente quando a correlação $r_{\hat{Y} Y}$ for muito alta (>98\%). Esta inconsistência ocorre pelo fato de o denominador da fórmula ser 2QMRes. Conforme proposto por Guimarães (1994) e por Leite \& Oliveira (2002), quando esta situação ocorre é necessário analisar graficamente a distribuição dos resíduos (Figura 3).

Ao analisar a Figura 3, verifica-se que as estimativas geradas pela equação 7 foram mais exatas, por se apresentarem com melhor e mais uniforme distribuição de resíduos que a equação 8 . Além disto, conforme dados do Quadro 7, a equação 7 resultou em menor erro médio porcentual (-1,62\% contra -2,34\%) e em menor $s_{\hat{Y} Y \%}$ $(7,74$ contra 8,66$)$. Tal fato indica que a equação 7 resulta em maior exatidão, devendo, portanto, ser escolhida. Então, devem ser adotados modelos volumétricos regionais, ou de dupla entrada, em inventários florestais, e a hipótese $H o_{(2)}$ é rejeitada.
As 50 parcelas do segundo povoamento tiveram o volume estimado pelas equações 7 e 8 , e na equação 7 utilizou-se a equação 9 para estimar $H t$ das árvores que não tiveram sua altura medida. Os resultados foram também comparados pelo procedimento de Leite \& Oliveira (2002), equação 8 versus equação 7, obtendose valores significativos para os testes $F$ e $t\left(128,451^{*}\right.$ e $\left.5,775^{*}\right)$, com uma correlação de $0,948>1-|\bar{e}|$. Assim, verifica-se que realmente as estimativas são diferentes.

Os intervalos de confiança para o total de madeira (em 67,81 ha do povoamento 1) estão apresentados no Quadro 8. Pode-se comprovar a maior exatidão das estimativas obtidas com o emprego de $V=f($ dap,$H t)$ com $H t=f(d a p, H d)$, uma vez que é o único método que atende ao limite de confiança (LC) obtido pelo valor real (método 1). Os demais métodos só atendem ao limite de confiança inferior.

Se os valores do Quadro 8 fossem considerados em um planejamento das atividades florestais, haveria a necessidade de alocar recursos materiais, monetários e de mão-de-obra (em maior número) com a equação 8 ,

Quadro 7 - Resultados do procedimento estatístico proposto por Leite \& Oliveira (2002) para avaliar a exatidão das estimativas oriundas das equações 7 e 8

Table 7 - Results of the statistical procedure proposed by Leite $\mathcal{E}$ Oliveira (2002) to evaluate the accuracy of the estimates originating from the equations 7 and 8

\begin{tabular}{|l|c|c|c|c|c|c|}
\hline \multicolumn{1}{|c|}{ Comparação } & $F$ & $t$ & $1-|\bar{e}|$ & $r_{\hat{Y} Y}$ & $s_{\hat{Y} Y}$ & EMP \\
\hline$H t_{\text {real }} \times$ Equação $7-V=f($ dap,$H d)$ & $11,906^{*}$ & $2,081^{\text {ns }}$ & 0,984 & 0,987 & 7,74 & $-1,62$ \\
$H t_{\text {real }} \times$ Equação $8-V=f($ dap $)$ & $39,151^{*}$ & $2,559^{n s}$ & 0,977 & 0,984 & 8,66 & $-2,34$ \\
\hline
\end{tabular}

ns $=$ não-significativo a $5 \% \mathrm{e}^{*}=$ significativo a $5 \%$ de probabilidade.

Equação 7 com equação 4

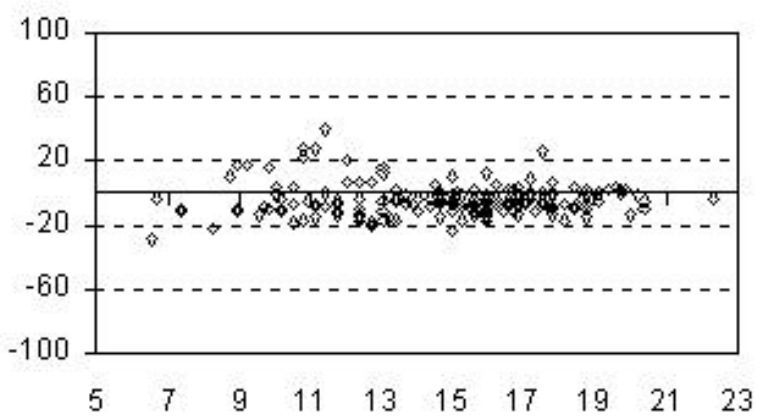

Equação 8

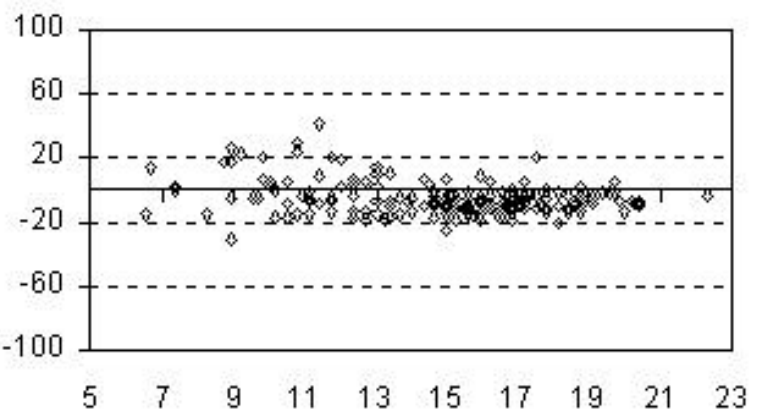

Figura 3 - Resíduos porcentuais em relação do dap obtidos pelas equações 7 e 8 .

Figure 3 - Percentage residues based on the dbh obtained by the equations 7 and 8 . 
Quadro 8 - Alguns resultados do inventário florestal, considerando as quatro parcelas do povoamento 1 Table 8 -Some results of the forestinventory, considering the 4 plots of stand 1

\begin{tabular}{|l|c|c|c|c|c|}
\hline \multicolumn{1}{|c|}{ Procedimento } & Método & Volume $\left(\mathrm{m}^{3} / \mathrm{ha}\right)$ & $\begin{array}{c}\mathrm{LC}_{\text {inferior }} \\
\left(\mathrm{m}^{3} / \mathrm{ha}\right)\end{array}$ & $\begin{array}{c}\text { Total }\left(\mathrm{m}^{3}\right) \\
\text { nas } \\
\left(\mathrm{m}^{3} / \mathrm{ha}\right)\end{array}$ \\
\hline Cubagem rigorosa de todas as árvores & 1 & $160,46(\mu)$ & $141,65\left(\mu-\frac{\sigma}{\sqrt{n}}\right)$ & $179,27\left(\mu+\frac{\sigma}{\sqrt{n}}\right)$ & $10881(Y)$ \\
\hline Modelo de Schumacker e Hall $\left(7 \mathrm{e} H t_{4}\right)$ & 2 & $156,27(\bar{V})$ & $135,00\left(\bar{V}-\frac{s_{\bar{V}}}{\sqrt{n}}\right)$ & $177,55\left(\bar{V}+\frac{s_{\bar{V}}}{\sqrt{n}}\right)$ & $10597(\hat{Y})$ \\
\hline Modelo de Schumacker e Hall $\left(7 \mathrm{e} H t_{6}\right)$ & 3 & $157,43(\bar{V})$ & $132,74\left(\bar{V}-\frac{s_{\bar{V}}}{\sqrt{n}}\right)$ & $182,12\left(\bar{V}+\frac{s_{\bar{V}}}{\sqrt{n}}\right)$ & $10675(\hat{Y})$ \\
\hline Modelo de BRENAC (8) & 4 & $152,67(\bar{V})$ & $133,99\left(\bar{V}-\frac{s_{\bar{V}}}{\sqrt{n}}\right)$ & $171,34\left(\bar{V}+\frac{s_{\bar{V}}}{\sqrt{n}}\right)$ & $10352(\hat{Y})$ \\
\hline
\end{tabular}

em comparação à equação 7. Por exemplo, em 67,81 ha, ao serem adotados os métodos 2 e 3 , haveria um erro de estimativa de $10.881-10.597=284 \mathrm{~m}^{3}$ e $10.881-10.675$ $=206 \mathrm{~m}^{3}$, respectivamente, contra $10.881-10.352=$ $529 \mathrm{~m}^{3}$ obtido pelo uso do método 4 (equação 8). Em termos porcentuais resultaria em -2,61 e -1,89\%, respectivamente, contra $-4,86 \%$ de diferença em relação ao total de madeira observado em 67,81 ha do povoamento. Embora sejam porcentuais relativamente baixos para um inventário florestal, em um exemplo hipotético, considerando uma área de 230 ha e mantendo-se os mesmos valores médios, haveria 964 e $697 \mathrm{~m}^{3}$ contra $1792 \mathrm{~m}^{3} \mathrm{de}$ erro na estimativa do volume total na área (230 ha).

Considerando o valor da madeira igual a $\mathrm{R} \$ 28 . \mathrm{m}^{-3}$, a diferença em 230 ha seria equivalente a [(1792-964)28] $=\mathrm{R} \$ 23.184,00$ para o uso do método 2 contra o método 4 , ou a $[(1.792-697) .28]=\mathrm{R} \$ 30.660,00$ para o uso do método 3 contra o método 4 . Desta maneira, indiretamente, pode-se provar que a hipótese $\mathrm{Ho}_{(2)}$ deve ser rejeitada, portanto devem ser adotados modelos regionais para quantificar o volume de madeira em maciços florestais. Podem, ainda, ser feitas inferências sobre o uso da equação hipsométrica mais adequada à totalização das parcelas do inventário, isto é, equação 4 ou 6.

Com o intuito de obter a melhor estimativa, deve-se adotar o método que proporcione o menor vício possível, ou seja, o método 3 é o mais adequado em inventários florestais. No exemplo hipotético descrito, esse método resulta em $-1,89 \%$ contra $-2,61 \%$ do método 2 ou $697 \mathrm{~m}^{3}$ contra $964 \mathrm{~m}^{3}$ de erro na estimativa do volume total na área (230 ha). Estes valores resultariam em uma diferença equivalente a $\mathrm{R} \$ 7.476,00$. Seria um valor baixo para ser utilizado no orçamento das atividades de treinamento da mão-de-obra, compra de veículos, combustível etc.

\section{CONCLUSÕES}

Os resultados mostraram ser mais adequado utilizar equações hipsométricas que tenham a altura dominante como uma das variáveis independentes, pois os resultados obtidos pelo modelo hipsométrico $\mathrm{Ln}\left(\mathrm{Ht}_{\mathrm{i}}\right)=$ $\beta_{0}+\beta_{1} \cdot\left(\text { dap }_{i}\right)^{-1}+\beta_{2} \cdot \operatorname{Ln}\left(H d_{i}\right)+\left(\varepsilon_{i}\right)$ demonstraram-se estatisticamente em níveis aceitáveis e contribuíram para o menor vício na variável volume. Modelos hipsométricos do tipo $H t=f($ dap $)$ devem ser adotados somente quando não forem detectadas variações na altura dominante das parcelas de um inventário florestal, uma vez que este tipo de relação hipsométrica, em cada parcela, se torna um processo moroso e, portanto, operacionalmente inadequado. Também, pode-se concluir que:

- o volume de árvores individuais não deve ser estimado apenas em função do dap;

- o volume de árvores individuais deve ser estimado em função do dap e da $H t$;

- a melhor alternativa para estimar a altura total em inventários florestais depende da heterogeneidade do povoamento e da intensidade de amostragem; e

R. Árvore, Viçosa-MG, v.27, n.3, p.301-310, 2003 
- o método selecionado em inventários florestais, que envolve uma relação hipsométrica, para quantificar o volume de madeira deve ser baseado, principalmente, no vício obtido pela variável de interesse, por exemplo, no volume, e não somente nos resultados obtidos pela equação hipsométrica testada.

\section{REFERÊNCIAS BIBLIOGRÁFICAS}

AVERY, T. E.; BURKHART, H. E. Forest measurements. 3.ed. New York: McGraw-Hill, 1983. 331 p.

BELCHIOR, P. R. M. Estimação de volumes total, de fuste e de galhos em mata secundária no município de Rio Vermelho. 1996. 75 f. Dissertação (Mestrado em Ciência Florestal) - Universidade Federal de Viçosa, Viçosa, 1996.

CAMPOS, J. C. C. A growth and yield study in thinned, even-aged stands of Pinus patula in Brazil. 1979. $129 \mathrm{f}$. Thesys (Doctor of Philosophy) - University of Washington, Washington, 1979.

CAMPOS, J. C. C; RIBEIRO, J. O.; PAULA NETO, F. Inventário florestal nacional, reflorestamento: Minas Gerais. Brasília, DF: IBDF, 1984. 126 p.

CAMPOS, A. L. A. S. Desenvolvimento de um sistema compatível de crescimento e produção para manejo de Eucalyptus grandis (W. Hill ex-Maiden). 1986. $73 \mathrm{f}$. Dissertação ( Mestrado em Ciência Florestal) Universidade Federal de Viçosa, Viçosa, 1986.

GRAYBILL, F. A. Theory and application of the linear model. Massachusetts: Duxbury Press, 1976. 704 p.
GUIMARÃES, D. P. Desenvolvimento de um modelo de distribuição diamétrica de passo invariante para prognose e projeção da estrutura de povoamentos de eucalipto. 1994. 160 f. Tese (Doutorado em Ciência Florestal) - Universidade Federal de Viçosa, Viçosa, 1994.

HUANG, S.; PRICE, D.; TITUS, S. J. Development of ecoregion-based height-diameter models for white spruce in boreal forests. Forest Ecology and Management, v. 129, p. $125-141,2000$.

HUSC, B.; MILLER, C. I.; BEERS, T. W. Forest mensuration. 2.ed. New York: Ronald Press, 1972. 409 p.

LEITE, H. G. Ajuste de um modelo de estimação de freqüência e produção por classe de diâmetro, para povoamentos de Eucalyptus saligna Smith. 1990. $78 \mathrm{f}$. Dissertação (Mestrado em Ciência Florestal) - Universidade Federal de Viçosa, 1990.

LEITE, H. G.; ANDRADE, V. C. L. Um método para condução de inventários florestais sem o uso de equações volumétricas. Revista Árvore, v. 26, n. 3, p. 321-328, 2002.

LEITE, H. G.; OLIVEIRA, F. H. T. Statistical procedure to test the identity of analytical methods. Communication Soil Science Plant Analitical, v. 33, p. 7 e 8, 2002.

REGAZZI, A. J.; LEITE, H. G. Análise de Regressão: teoria e aplicações em manejo florestal. Viçosa: UFV, 1992. 236 p. não publicado.

SCHUMACHER, F. X.; HALL, F. S. Logarithmic expression of the timber volume. Journal of Agriculture Research, v. 47, n. 9, p. 719-34, 1933. 